\title{
New enhanced and effective method for staging cancer to detect lymph nodes after fat-dissociation
}

\author{
SHIKI FUJINO $^{1}$, NORIKATSU MIYOSHI ${ }^{1}$, MASAYUKI OHUE ${ }^{1}$, SHINGO NOURA ${ }^{1}$, \\ YASUHIKO TOMITA $^{2}$, MASAHIKO YANO ${ }^{1}$ and MASATO SAKON ${ }^{1}$ \\ Departments of ${ }^{1}$ Surgery and ${ }^{2}$ Pathology, Osaka Medical Center for Cancer and \\ Cardiovascular Diseases, Osaka 537-8511, Japan
}

Received February 17, 2014; Accepted May 2, 2014

DOI: $10.3892 /$ or.2014.3315

\begin{abstract}
Pathologic staging is a key factor in the treatment and outcome of cancer patients, and lymph node status plays a pivotal role in this staging process. Several methods of lymph node assessment, such as the fat clearance, Schwartz solution and GEWF solution, are unpopular as they require a significant amount of time (1-9 days) to clear the fat of the resected mesentery and to visualize the lymph nodes. In the present study, we discussed a more simple and effective method, called the fat-dissociation method, which clears the fat using enzymes and allows for easy identification of lymph nodes around vessels. This new procedure facilitates precise staging of cancer which directs the post-operative treatment plan.
\end{abstract}

\section{Introduction}

Cancer is a leading cause of mortality worldwide and the number of cancer cases and deaths is projected to continue rising, with the current estimation at 17 million deaths due to cancer per year (1). Colon, lung, breast, liver and stomach cancer have the highest mortality rates. Specifically, colorectal cancer (CRC) is the leading cause of mortality among women and the third leading cause among men in Japan, as of 2011, and it continues to increase (2). Surgical resection of a primary tumor and regional lymph nodes is important for CRC treatment, and the pathological staging, represented as $\mathrm{T}$ and $\mathrm{N}$ factors, depends on tumor invasion and lymph node metastasis, respectively, in the surgically-resected specimen. There are several reports regarding CRC prognosis and pathological factors, and $\mathrm{N}$ factor (lymph node metastasis) and the number of the nodes are reported to be one of the most important prognostic factors $(3,4)$. It is necessary and essential to evaluate the total number of resected lymph nodes and metastases to

Correspondence to: Dr Norikatsu Miyoshi, Department of Surgery, Osaka Medical Center for Cancer and Cardiovascular Diseases, 1-3-3 Nakamichi, Higashinari-Ku, Osaka 537-8511, Japan E-mail: miyosi-no@mc.pref.osaka.jp

Key words: lymph nodes, colorectal cancer, staging, fat-dissociation determine the adjuvant chemotherapy regimen (4). In relation to the surgically-resected lymph nodes in CRC, the National Comprehensive Cancer network recommends the examination of at least 12 lymph nodes for reliable CRC staging (5).

Various methods to determine sufficient number of surgically-resected lymph nodes, such as fat clearance $(6,7)$, Schwartz (8) and GEWF solution $(9,10)$, have been reported. However, a caveat of these methods is that it requires a significant amount of time (1-9 days) to clear the fat from the resected mesentery in which there are lymph nodes to be evaluated. There is presently no consensus on a convenient and effective method to detect the lymph nodes. According to the NCCN and ESMO guidelines, the Japanese Society for Cancer of the Colon and Rectum also recommends identifying at least 12 lymph nodes for appropriate CRC staging $(5,11)$. The general and traditional method is to manually search for lymph nodes by touching then fixing with formalin. Although the surgeon can efficiently evaluate the lymph nodes and metastases, the palpation method is still very time-consuming. In the present study, we present a simple and effective method, called as the fat-dissociation method, which dissociates the mesenteric fat therefore reducing the volume and leading to clear visualization of these lymph nodes. This method allows for faster and easier identification of lymph nodes compared to the conventional palpation method, specifically the count and condition of the extracted lymph nodes.

\section{Materials and methods}

Clinical tissue samples. The present study included 19 patients who underwent surgery for CRC at Osaka Medical Center for Cancer and Cardiovascular Diseases (OMCCCD) from November to December 2013. This study was performed after written informed consent had been obtained in accordance with our institutional ethics guidelines approved by the OMCCCD Ethics Committee. The resected tumor and surrounding tissue were examined. The general course to examine the resected specimen is as follows: the mesentery is carefully separated from the primary tumor lesion and the surgeons explore the lymph nodes in the mesentery, which takes $30 \mathrm{~min}$ to $1 \mathrm{~h}$. In the present study, we also performed the general procedure above, and re-examined the lymph nodes remaining in the mesentery that had already been explored (Fig. 1). After the 


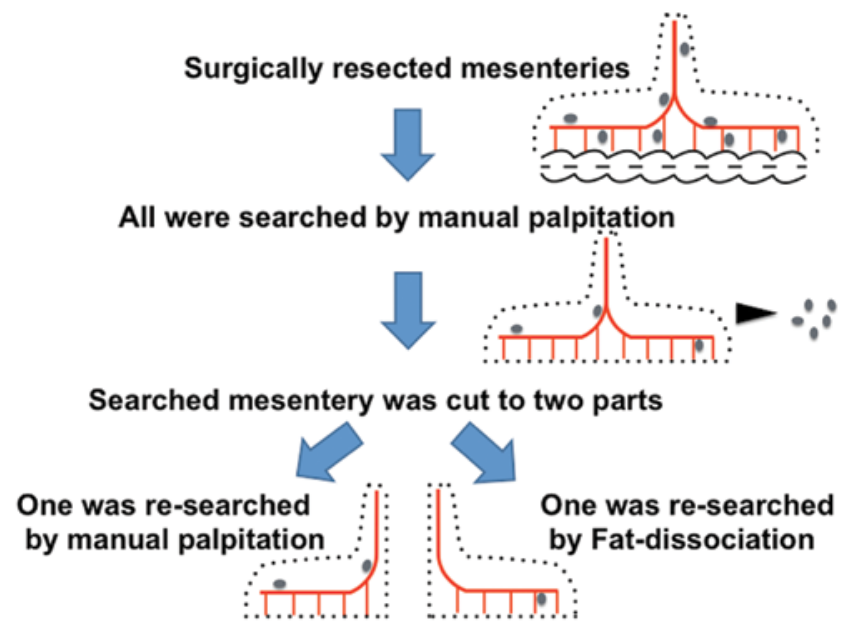

Figure 1. The whole process of the preparation and examination of lymph nodes in the surgically-resected specimen. All mesenteries were surgically resected from CRC patients. First, lymph nodes were searched for by surgeons by the conventional palpation method. Second, the remaining mesentery already examined was cut into two parts of equal mass (calculated by the weight). One part was re-searched for lymph nodes by two other surgeons by the palpation method for at least $10 \mathrm{~min}$ and the other part was examined by the fat-dissociation method. CRC, colorectal cancer.

conventional method, namely, searching for lymph nodes in the mesentery by a surgeon palpating manually, the remaining mesenteric specimen was cut into two parts of equal mass, and one part was re-searched to examine the remaining lymph nodes by two other surgeons palpating manually for at least $10 \mathrm{~min}$. The other piece of mesenteric specimen was examined for lymph nodes by using the new method of dissociating the mesenteric fat to visualize the lymph nodes clearly. All picked up lymph nodes were treated with standard formalin fixation and evaluated by using hematoxylin and eosin staining.

Dissociation of mesenteric tissue. The mesenteric tissue was dissociated by using the following solution: $0.5-1 \mathrm{mg} / \mathrm{ml}$ collagenase (C6885; Sigma-Aldrich, St. Louis, MO, USA) with up to $0.25 \%$ trypsin (25200072; Life Technologies, Carlsbad, CA, USA). We examined the dilution effect in DPBS (14190250; Life Technologies) or DMEM (D5796; SigmaAldrich), but there was no significant difference; finally, the solution was diluted in DPBS. Regarding the volume of the solution added to the specimen, we investigated several conditions and finally $1 \mathrm{ml}$ solution was added to a $3-\mathrm{g}$ piece of

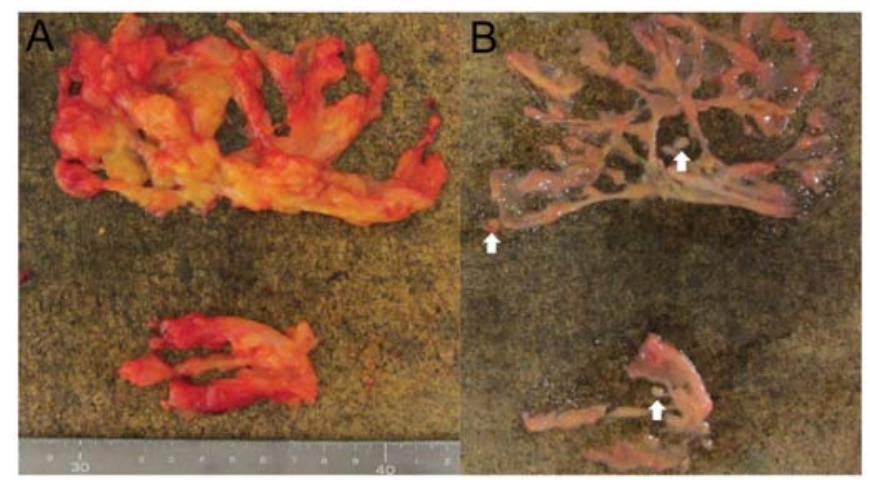

Figure 3. Images of the surgically resected mesentery before/after the fatdissociation. (A) The mesenteric fat was examined after manual examination (by the palpation of the lymph nodes). (B) After fat-dissociation, the mesenteric fat was dissociated, and vessel structures and lymph nodes (arrows) were visualized.

mesentery. The components were mixed at $100 \mathrm{rpm}$ using a rotator for $10 \mathrm{~min}$ at several temperatures (Fig. 2). Next, the mesentery was placed on a paper to collect the dissociated fat tissue, followed by grinding on the paper. The lymph nodes were then easily identified since the area around the vessels was clear of fat, hence the naming of this new methodology as the fat-dissociation method (Fig. 3).

Statistical analysis. For continuous variables, data are expressed as median (range). The relationship between the lymph node count by re-palpation and the fat-dissociation method was analyzed using the Wilcoxon rank-sum and signed-rank tests. They were analyzed using JMP software (version 11.0; SAS Institute, Cary, NC, USA). Differences with p-value $<0.05$ were considered statistically significant.

\section{Results}

First, several conditions of fat-dissociation were examined whether the mesenteric fat were dissociated (Table I). We tested various concentrations and mixtures of reagents to establish the ideal and practical concentration, incubation time, and temperature. When only collagenase or trypsin was used, the dissociation effect was moderate. The most effective and applicable condition (and the condition used in this report) was $1 \mathrm{mg} / \mathrm{ml}$ collagenase and $0.25 \%$ trypsin for $10 \mathrm{~min}$ at $40^{\circ} \mathrm{C}$.

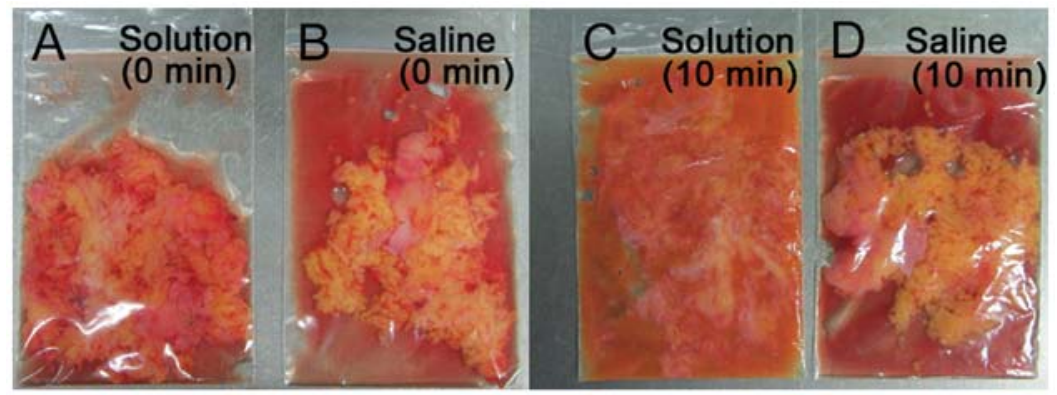

Figure 2. Images during the course of the fat-dissociation method. The mesenteric tissue with (A) the fat-dissociation solution or (control, B) saline. After $10 \mathrm{~min}$, the mesenteric fat was dissociated with $(\mathrm{C})$ the fat-dissociation solution compared to the (D) saline. 
Table I. Conditions of the fat-dissociation method.

\begin{tabular}{lcccc}
\hline $\begin{array}{l}\text { Collagenase } \\
(\mathrm{mg} / \mathrm{ml})\end{array}$ & $\begin{array}{c}\text { Trypsin } \\
(\%)\end{array}$ & $\begin{array}{c}\text { Incubation } \\
\text { time }(\mathrm{min})\end{array}$ & $\begin{array}{c}\text { Temp. } \\
\left({ }^{\circ} \mathrm{C}\right)\end{array}$ & Dissociation $^{\mathrm{a}}$ \\
\hline 0 & 0.25 & 120 & 40 & 2 \\
0.5 & 0.05 & 30 & 22 & 1 \\
0.5 & 0.125 & 45 & 37 & 3 \\
0.5 & 0.125 & $7200^{\mathrm{b}}$ & 22 & 3 \\
1 & 0 & 30 & 22 & 2 \\
1 & 0.25 & 360 & 22 & 3 \\
1 & 0.25 & 15 & 37 & 3 \\
1 & 0.25 & 10 & 40 & 3 \\
\hline
\end{tabular}

aEstimation of dissociation: 1, weak; 2, moderate; 3, good. Temp., temperature. ${ }^{\mathrm{b}} 7200 \mathrm{~min}$ are equal to $12 \mathrm{~h}$.

The defined lymph node dissection was performed for each CRC case according to the JSCCR guidelines (12). A total of 20 specimens derived from 19 CRC cases were evaluated on the number of lymph nodes in the first and second lymph node regions by pathological examination according to the Japanese Classification of Colorectal Carcinoma (13)
Table III. Median number of lymph nodes by each method.

\begin{tabular}{lrrr}
\hline & $\begin{array}{c}\text { Palpation } \\
\text { method }\end{array}$ & $\begin{array}{c}\text { Re-palpation } \\
\text { method }\end{array}$ & $\begin{array}{r}\text { Fat-dissociation } \\
\text { method }\end{array}$ \\
\hline $\begin{array}{l}\text { 1st LNs } \\
\text { (range) }\end{array}$ & 8 & 0.5 & 2 \\
2nd LNs & $(3-23)$ & $(0-3)$ & $(0-8)$ \\
(range) & $(0-15)$ & $(0-3)$ & 1 \\
Total LNs & 11.5 & 1 & $(0-4)$ \\
(range) & $(3-33)$ & $(0-6)$ & $(1-11)$ \\
\hline
\end{tabular}

LNs, lymph nodes.

(summarized in Table II). The median number of lymph nodes found by the traditional palpation method was eight (range, 3-23) and four (range, 0-15) in the first and second lymph node regions, respectively. Next, the median number of lymph nodes searched by the re-palpation method was 0.5 (range, $0-3$ ) in the first lymph node region and 0 (range, 0-3) in the second lymph node region. Finally, the median count examined by the fat-dissociation method was two (range, 0-8) in the first

Table II. Summary of 19 patients.

\begin{tabular}{c} 
Positive/total lymph nodes \\
\cline { 2 - 5 } Age
\end{tabular}

Case Gender (years) BMI Operation Palpation palpation dissociation Palpation palpation dissociation $\mathrm{T} N$ Stage

\begin{tabular}{|c|c|c|c|c|c|c|c|c|c|c|c|c|c|}
\hline 1 & $\mathrm{M}$ & 81 & 23.2 & $\mathrm{~S}$ & $0 / 4$ & $0 / 0$ & $0 / 2$ & $0 / 4$ & $0 / 3$ & $0 / 0$ & T1b & No & I \\
\hline 2 & $\mathrm{~F}$ & 75 & 21.8 & $\mathrm{~L}$ & $0 / 5$ & $0 / 0$ & $0 / 0$ & $0 / 2$ & $0 / 0$ & $0 / 1$ & $\mathrm{~T} 2$ & No & I \\
\hline 3 & $\mathrm{~F}$ & 63 & 21.6 & LAR & $0 / 6$ & $0 / 0$ & $0 / 0$ & $0 / 5$ & $0 / 0$ & $0 / 1$ & $\mathrm{~T} 2$ & No & I \\
\hline 4 & M & 62 & 21.8 & LAR & $0 / 7$ & $0 / 1$ & $0 / 6$ & $0 / 2$ & $0 / 1$ & $0 / 2$ & T3 & No & II \\
\hline 5 & M & 65 & 23.3 & LAR & $0 / 14$ & $0 / 0$ & $0 / 0$ & $0 / 5$ & $0 / 0$ & $0 / 1$ & $\mathrm{~T} 3$ & No & II \\
\hline 6 & $\mathrm{~F}$ & 67 & 20.8 & LAR & $0 / 14$ & $0 / 1$ & $0 / 8$ & $0 / 3$ & $0 / 0$ & $0 / 3$ & T3 & N0 & II \\
\hline 7 & M & 76 & 18.7 & $\mathrm{~T}$ & $0 / 7$ & $0 / 0$ & $0 / 3$ & $0 / 0$ & $0 / 0$ & $0 / 0$ & $\mathrm{~T} 3$ & N0 & II \\
\hline 7 & M & 76 & 18.7 & APR & $0 / 10$ & $0 / 1$ & $0 / 2$ & $0 / 4$ & $0 / 1$ & $0 / 0$ & $\mathrm{~T} 4 \mathrm{~b}$ & NO & II \\
\hline 8 & $\mathrm{~F}$ & 67 & 22.5 & sLAR & $3 / 8$ & $0 / 0$ & $0 / 2$ & $0 / 1$ & $0 / 0$ & $0 / 2$ & $\mathrm{~T} 3$ & N1 & IIIa \\
\hline 9 & $\mathrm{~F}$ & 70 & 25.1 & $\mathrm{~S}$ & $3 / 3$ & $0 / 0$ & $0 / 2$ & $0 / 0$ & $0 / 0$ & $0 / 2$ & $\mathrm{~T} 2$ & N1 & IIIa \\
\hline 10 & M & 74 & 18.7 & $\mathrm{~S}$ & $0 / 21$ & $0 / 3$ & $0 / 7$ & $0 / 12$ & $0 / 3$ & $0 / 3$ & T3 & N1 & IIIa \\
\hline 11 & M & 82 & 18.5 & $\mathrm{~S}$ & $1 / 23$ & $0 / 1$ & $0 / 2$ & $0 / 4$ & $0 / 0$ & $0 / 1$ & $\mathrm{~T} 3$ & N1 & IIIa \\
\hline 12 & M & 68 & 22.9 & AR & $1 / 5$ & $0 / 1$ & $0 / 1$ & $0 / 3$ & $0 / 0$ & $0 / 3$ & $\mathrm{~T} 4 \mathrm{a}$ & N1 & IIIa \\
\hline 13 & $\mathrm{~F}$ & 77 & 17.6 & sLAR & $3 / 19$ & $0 / 0$ & $0 / 3$ & $0 / 5$ & $0 / 0$ & $0 / 1$ & $\mathrm{~T} 3$ & N1 & IIIa \\
\hline 14 & $\mathrm{~F}$ & 71 & 23.6 & $\mathrm{~L}$ & $1 / 7$ & $0 / 1$ & $0 / 1$ & $0 / 5$ & $0 / 1$ & $0 / 0$ & $\mathrm{~T} 1 \mathrm{~b}$ & N1 & IIIa \\
\hline 15 & M & 57 & 22.7 & $\mathrm{R}$ & $3 / 8$ & $0 / 1$ & $0 / 2$ & $0 / 2$ & $0 / 1$ & $0 / 4$ & $\mathrm{~T} 4 \mathrm{a}$ & N1 & IIIa \\
\hline 16 & $\mathrm{~F}$ & 60 & 24.2 & LAR & $3 / 19$ & $0 / 2$ & $0 / 1$ & $0 / 8$ & $0 / 0$ & $0 / 1$ & $\mathrm{~T} 3$ & N1 & IIIa \\
\hline 17 & $\mathrm{~F}$ & 57 & 29.8 & $\mathrm{~L}$ & $4 / 16$ & $0 / 0$ & $0 / 0$ & $0 / 6$ & $0 / 0$ & $0 / 1$ & $\mathrm{~T} 2$ & N2 & IIIb \\
\hline 18 & M & 71 & 21.4 & AR & $5 / 6$ & $1 / 1$ & $2 / 3$ & $1 / 2$ & $0 / 0$ & $1 / 1$ & $\mathrm{~T} 4 \mathrm{a}$ & N2 & IV \\
\hline 19 & $\mathrm{~F}$ & 69 & 25.0 & LAR & $5 / 10$ & $0 / 0$ & $0 / 2$ & $1 / 15$ & $0 / 1$ & $0 / 2$ & T3 & N2 & IV \\
\hline
\end{tabular}

S, sigmoid colectomy; L, left colectomy; LAR, low anterior resection; T, transverse colectomy; APR, abdominoperineal resection; sLAR, super low anterior resection; $\mathrm{AR}$, anterior resection; $\mathrm{R}$, right colectomy. $\mathrm{T}$ and $\mathrm{N}$ factors and stages are according to the Japanese Classification of Colorectal Carcinoma. T, primary tumor; $\mathrm{N}$, regional lymph nodes. 


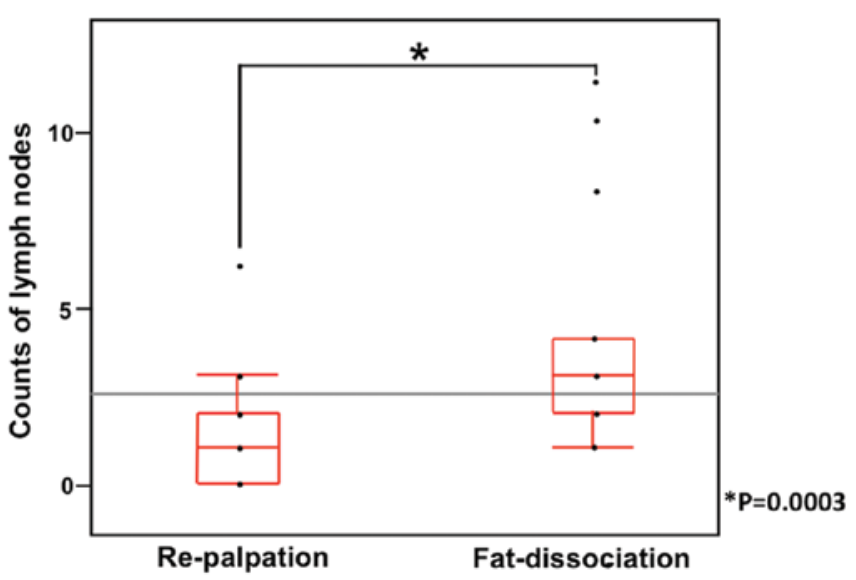

Figure 4. Analysis of the evaluated number of lymph nodes of the re-palpation and the fat-dissociation method. The number of total lymph nodes (1st and 2nd regional lymph nodes) of the re-palpation and the fat-dissociation method was analyzed. There was a significant increase in the number of lymph nodes found by the fat-dissociation method compared to the re-palpation method (Wilcoxon rank-sum test, $\mathrm{P}=0.0003$ ).

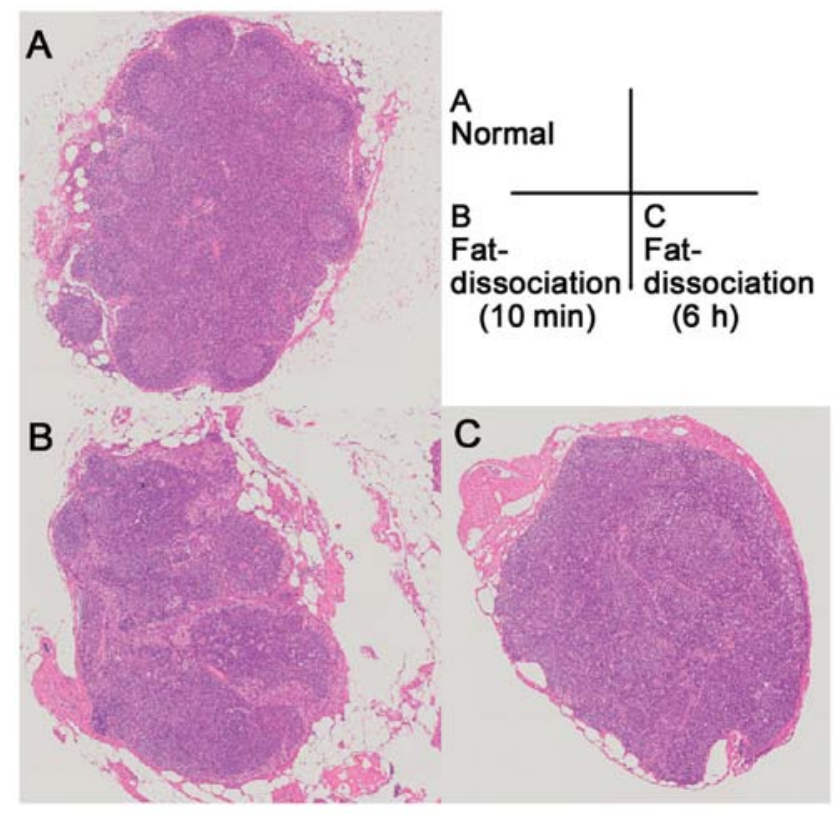

Figure 5. Pathological examination of lymph nodes by the conventional or the new method. (A) By the traditional palpation method, the lymph node was surrounded by fat. (B) By the fat-dissociation method, there were some fat tissues around the lymph node. The structure of the lymph node was preserved. (C) By the modified fat-dissociation method, the lymph node was not covered with fat tissue; however, the structure of the lymph node was still completely preserved.

lymph node region and one (range, $0-4$ ) in the second lymph node region (Table III). The use of the fat-dissociation method resulted in a significant increase in the total number of lymph nodes found compared to the traditional method (Fig. 4). The Wilcoxon singed-rank test showed the significant difference between the fat-dissociation and re-palpation methods $(\mathrm{P}=0.0003)$.

The lymph nodes were all pathologically examined, and those explored by the fat-dissociation method were examined without any difficulties (Fig. 5).

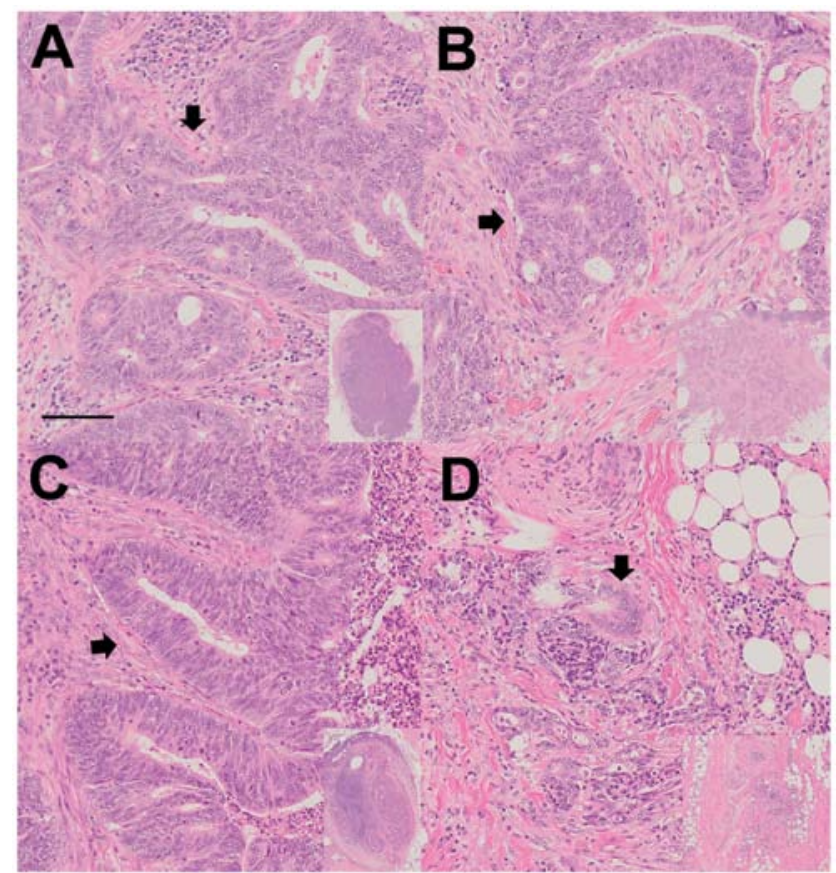

Figure 6. Pathological findings of lymph node metastasis. Lymph node metastases searched by (A) conventional palpation or (B) fat-dissociation method and tumor nodule searched by (C) palpation or (D) fat-dissociation method was evaluated (arrows). (Scale bar, $100 \mu \mathrm{m})$.

\section{Discussion}

The fat-dissociation method reported is effective and applicable to searching for lymph nodes in the surgically-resected mesentery. In the present study, upon evaluation of the time required for the traditional palpation (median, $50 \mathrm{~min}$ ) and the fat-dissociation method (median, $18 \mathrm{~min}$ ), it was revealed that more time is required to search for lymph nodes in the mesenteric fat by the palpation method. The fat-dissociation method can save time on lymph node examination and exploration. Generally, searching for lymph nodes around vessels is difficult as surrounding vascular sheaths and nerves are fixed and hard to analyze carefully. The fat-dissociation method can change the mesenteric structure, making the mesenteric fat soft and reducing the volume, which results in easier examination of the lymph nodes around vessels. In our previous study, collagenase and trypsin were used to generate primary culture from surgically-resected specimens, generating in vitro culture of the clinical samples $(14,15)$. The fat-dissociation method requires only $10 \mathrm{~min}$ to clear the mesenteric fat, resulting in visualization of the lymph nodes. In routine study, short incubation times may be risky, therefore we also evaluated whether the incubation time can be extended. When we used the solution at half its concentration and at several temperatures, the mesenteric fat dissociated in $45 \mathrm{~min}$, and even after $12 \mathrm{~h}$, the lymph nodes kept stable. This indicates that the half-concentration solution would be feasible and applicable in several institutions. We also evaluated the lymph node metastases. In case 18 , lymph node metastases were diagnosed after the fat-dissociation method as well as the normal conventional palpation method (Fig. 6). In the Japanese classification of CRC, tumor nodule is defined as lymph node metastasis (13). 
Tumor nodule exists separately from intestine without lymph node structure. We were able to evaluate the tumor nodule and there were no differences of pathological findings between these two methods.

The patients of CRC stage III and high risk stage II are recommended to receive adjuvant chemotherapy after surgery (16-21); hence, adequate searching for lymph nodes is necessary and important. The impact of the fat-dissociation method is in enabling all clinicians to examine the lymph nodes easily and effectively by modifying the condition of the solution according to their individual work style.

In conclusion, we described a novel and effective technique to dissociate the mesenteric fat, resulting in easier examination of the lymph nodes. This new method, called the fat-dissociation method, can visualize lymph nodes easily and effectively in surgically-resected mesentery compared to the conventional palpation method.

\section{Acknowledgements}

The authors thank Dr T. Fukata, Dr T. Umeda and Dr T. Hara for searching for lymph nodes in the surgically-resected mesentery.

\section{References}

1. Thun MJ, DeLancey JO, Center MM, Jemal A and Ward EM The global burden of cancer: priorities for prevention. Carcinogenesis 31: 100-110, 2010.

2. Center for Cancer Control and Information Services NCC: Japan recent cancer statistics, 2011. http://ganjoho.jp/public/statistics/pub/statistics01.html. Accessed December 30, 2013.

3. Van Cutsem E, Nordlinger B, Cervantes A; ESMO Guidelines Working Group: Advanced colorectal cancer: ESMO Clinical Practice Guidelines for treatment. Ann Oncol 21 (Suppl 5): v93-v97, 2010.

4. Labianca R, Nordlinger B, Beretta GD, et al: Early colon cancer: ESMO Clinical Practice Guidelines for diagnosis, treatment and follow-up. Ann Oncol 24 (Suppl 6): vi64-vi72, 2013.

5. Network NCC NCCN guidlines for treatment of cancer by site: Colon/Rectal Cancer. http://www.nccn.org/professionals/ physician_gls/f guidelines.asp. Accessed December 30, 2013.

6. Hida J, Mori N, Kubo R, et al: Metastases from carcinoma of the colon and rectum detected in small lymph nodes by the clearing method. J Am Coll Surg 178: 223-228, 1994.
7. Morikawa E, Yasutomi M, Shindou K, et al: Distribution of metastatic lymph nodes in colorectal cancer by the modified clearing method. Dis Colon Rectum 37: 219-223, 1994.

8. Chapman B, Paquette C, Tooke C, et al: Impact of Schwartz enhanced visualization solution on staging colorectal cancer and clinicopathological features associated with lymph node count. Dis Colon Rectum 56: 1028-1035, 2013.

9. Gregurek SF and Wu HH: Can GEWF solution improve the retrieval of lymph nodes from colorectal cancer resections? Arch Pathol Lab Med 133: 83-86, 2009.

10. Newell KJ, Sawka BW, Rudrick BF and Driman DK: GEWF solution. Arch Pathol Lab Med 125: 642-645, 2001.

11. ESMO Oncology Clinical Practice Guidlines. http://www.esmo. org/Guidelines-Practice/Clinical-Practice-Guidelines. Accessed December 30, 2013.

12. Japanese Society for Cancer of the Colon and Rectum. JSCCR Guidelines 2010 for the Treatment of Colorectal Cancer. Kanehara, Tokyo, pp13-15, 2010.

13. Japanese Society for Cancer of the Colon and Rectum. Japanese Classification of Colorectal Carcinoma. 8th edition. Kanehara, Tokyo, pp11-14, 2013.

14. Miyoshi N, Ishii H, Nagai K, et al: Defined factors induce reprogramming of gastrointestinal cancer cells. Proc Natl Acad Sci USA 107: 40-45, 2010.

15. Miyoshi N, Ishii H, Nagano H, et al: Reprogramming of mouse and human cells to pluripotency using mature microRNAs. Cell Stem Cell 8: 633-638, 2011.

16. Figueredo A, Charette ML, Maroun J, Brouwers MC and Zuraw L: Adjuvant therapy for stage II colon cancer: a systematic review from the Cancer Care Ontario Program in evidence-based care's gastrointestinal cancer disease site group. J Clin Oncol 22: 3395-3407, 2004.

17. Gill S, Loprinzi CL, Sargent DJ, et al: Pooled analysis of fluorouracil-based adjuvant therapy for stage II and III colon cancer: who benefits and by how much? J Clin Oncol 22: 1797-1806, 2004.

18. Benson AB III, Schrag D, Somerfield MR, et al: American Society of Clinical Oncology recommendations on adjuvant chemotherapy for stage II colon cancer. J Clin Oncol 22: 3408-3419, 2004.

19. André T, Boni C, Mounedji-Boudiaf L, et al: Oxaliplatin, fluorouracil, and leucovorin as adjuvant treatment for colon cancer. New Engl J Med 350: 2343-2351, 2004.

20. André T, Boni C, Navarro M, et al: Improved overall survival with oxaliplatin, fluorouracil, and leucovorin as adjuvant treatment in stage II or III colon cancer in the MOSAIC trial. J Clin Oncol 27: 3109-3116, 2009.

21. Kuebler JP, Wieand HS, O'Connell MJ, et al: Oxaliplatin combined with weekly bolus fluorouracil and leucovorin as surgical adjuvant chemotherapy for stage II and III colon cancer: results from NSABP C-07. J Clin Oncol 25: 2198-2204, 2007. 